\title{
Quantitative Measurement of Muscle Atrophy and Fat Infiltration of the Supraspinatus Muscle Using Ultrasonography After Arthroscopic Rotator Cuff Repair
}

\author{
Yong Ki Kim, $\mathrm{MD}^{1}$, Eun Seok Choi, $\mathrm{MD}, \mathrm{PhD}^{1}$, Keon Tae Kim, $\mathrm{MD}^{2}$, \\ Jung Ro Yoon, $\mathrm{MD}^{2}$, Sang Han Chae, $\mathrm{MD}^{3}$
}

\begin{abstract}
${ }^{1}$ Department of Rehabilitation Medicine, Daejeon St. Mary's Hospital, College of Medicine, The Catholic University of Korea, Daejeon; ${ }^{2}$ Department of Rehabilitation Medicine, St. Paul's Hospital, College of Medicine, The Catholic University of Korea, Seoul; ${ }^{3}$ Department of Rehabilitation Medicine, Mediplex Sejong Hospital, Incheon, Korea
\end{abstract}

Objective To investigate the utility of ultrasonography to objectively examine morphological changes (i.e., muscle atrophy and fatty infiltration) of the supraspinatus muscle.

Methods Thirty-four patients were prospectively enrolled in this study. The degrees of muscle atrophy and fat infiltration were measured using ultrasonography 3-4 months after arthroscopic supraspinatus tendon repair. Shoulder function (i.e., shoulder active range of motion, visual analogue scale, and constant score) was examined. Using the symmetricity of the muscles in the human body, the degrees of morphological changes of the supraspinatus muscle were quantitatively measured. The associations between the morphological changes of the supraspinatus muscle and shoulder function were identified.

Results There were statistically significant differences in the cross-sectional area (CSA) and echogenicity between the surgery and non-surgery sides $(\mathrm{p}<0.001)$. The CSA ratio, which represents the degree of muscle atrophy, was associated with shoulder forward flexion, external rotation, and constant score; however, the echogenicity ratio, which represents the degree of fat infiltration, was not associated with shoulder function after surgery.

Conclusion This study demonstrated that shoulder function could be predicted by evaluating the morphological changes of the supraspinatus muscle using ultrasonography and that objective evaluation is possible through quantitative measurement using the symmetricity of the human body.

Keywords Rotator cuff injuries, Ultrasonography, Supraspinatus, Atrophy, Muscles

Received May 16, 2017; Accepted August 14, 2017

Corresponding author: Sang Han Chae

Department of Rehabilitation Medicine, Mediplex Sejong Hospital, 20 Gyeyangmunhwa-ro, Gyeyang-gu, Incheon 21080, Korea. Tel: +82-32-240-8000, Fax: +82-32-240-8899, E-mail: shchae2@hanmail.net

ORCID: Yong Ki Kim (https://orcid.org/0000-0002-2116-0500); Eun Seok Choi (https://orcid.org/0000-0001-6073-248X);Keon Tae Kim (https://orcid. org/0000-0001-9174-909X); Jung Ro Yoon (https://orcid.org/0000-0002-3323-3738); Sang Han Chae (https://orcid.org/0000-0003-2436-0425).

(c) This is an open-access article distributed under the terms of the Creative Commons Attribution Non-Commercial License (http://creativecommons.org/ licenses/by-nc/4.0) which permits unrestricted noncommercial use, distribution, and reproduction in any medium, provided the original work is properly cited. Copyright $\odot 2018$ by Korean Academy of Rehabilitation Medicine 


\section{INTRODUCTION}

A shoulder rotator cuff tear (RCT) is the most common cause of shoulder pain. There are many treatment options for a RCT, including conservative and surgical treatments. Treatment of a RCT is performed based on patient-related factors such as age, amount of activity, and degree of tendon damage [1]. Among the treatment options, arthroscopic rotator cuff repair has been frequently performed because it is associated with few postoperative complications and rapid recovery [2-4]. Many studies have reported that morphological changes of muscle, such as preoperative muscle atrophy and fat infiltration, are associated with shoulder function and can be used as a predictor of postoperative prognosis [5-7]. However, few studies have evaluated the associations between postoperative morphologic changes of the muscle and shoulder function. Additionally, most studies that evaluated morphological changes of the muscle used magnetic resonance imaging (MRI) or computed tomography (CT). The results of recent studies that evaluated morphological changes of the muscle using ultrasonography, however, were not significantly different from those of studies using MRI or CT [8-10]. Assessments that use ultrasonography have advantages over assessments that use MRI in terms of cost and time. An ultrasonography assessment also has the advantage of not exposing patients to radiation, unlike CT. Recent studies regarding the morphological changes of muscle using ultrasonography, however, the assessment of the morphological changes of the muscle also had disadvantages. For example, there was no exact reference value because the morphological changes of the muscle were evaluated subjectively by dividing the grade of the muscle condition or based on the peripheral fat tissues or surrounding muscles [8-10]. Studies that evaluated the dominant and non-dominant side of human muscles through ultrasonography have shown symmetricity of the muscles of the human body $[11,12]$. Therefore, if quantitative evaluation is performed based on the same site, but on the opposite side, by using the symmetricity of the human body, objective evaluation of the muscle morphology may be possible. The purpose of this study was to investigate the utility of ultrasonography to examine objectively the morphological changes of the supraspinatus muscle using the symmetricity of the human body. The cross-sectional area (CSA; i.e., assessment of muscle atrophy) and echogenicity (i.e., assessment of fatty infiltration) of the supraspinatus muscles on the surgery and the non-surgery sides of patients who underwent arthroscopic supraspinatus tendon repair were measured using ultrasonography. Using the symmetricity of muscles of the human body, the degrees of muscle atrophy and fatty infiltration were quantitatively calculated by obtaining the ratio of the surgery side based on the non-surgery side. Associations between the morphological changes of the supraspinatus muscle and shoulder function were also identified.

\section{MATERIALS AND METHODS}

Patients visited the Department of Rehabilitation Medicine after undergoing arthroscopic supraspinatus tendon repair. The degrees of muscle atrophy and fat infiltration were measured using ultrasonography 3-4 months after arthroscopic supraspinatus tendon repair. The degree of muscle atrophy was evaluated by measuring the CSA of the supraspinatus muscle; the degree of fat infiltration was evaluated by measuring the mean echogenicity (since muscle atrophy progresses, the value of the CSA becomes smaller; as fat infiltration progresses, the value of the echogenicity becomes larger) [13]. The ratio of the CSA and echogenicity of the surgery side based on the non-surgery side was obtained using the symmetricity of the human body to quantitatively evaluate the degrees of muscle atrophy and fat infiltration. Although the human body is symmetrical, the muscle on the non-dominant side may be smaller, and muscle atrophy may occur on the surgery side due to disuse after surgery. If patients who had undergone surgery on the non-dominant side were included in this study, then it would have been difficult to accurately determine if the muscle was smaller because it was on the non-dominant side or because the size of the muscle had been reduced due to disuse after surgery. Therefore, only patients who had undergone surgery on the dominant side were included in this study. This study was conducted in accordance with the 1964 Declaration of Helsinki and written informed consent was obtained from all patients. This study was approved by the Institutional Review Board of Catholic University of Korea Daejeon St. Mary's Hospital (No. DC16-RISI 0016). 


\section{Participants}

Thirty-four patients were prospectively enrolled in this study ( 22 women and 12 men; age, 59.1 \pm 7.4 years; range, 46-78 years; postoperative day, 97.1 \pm 7.7 ) (Table $1)$. The rotator cuff repair surgeries were performed by the same orthopedic surgeon using the suture bridge repair technique by arthroscopy. The patients visited the Department of Rehabilitation Medicine 1 month after surgery and were educated regarding scapular-stabilizing exercises and shoulder range of motion (ROM) exercises, which they could perform independently at home. They also received physical therapy for shoulder pain and shoulder-passive ROM exercises under the guidance of a physical therapist 2-3 times per week. They visited the outpatient clinic of the Department of Rehabilitation Medicine again 3-4 months after surgery for ultrasonography evaluation. The inclusion criteria were as follows: patients who had undergone surgery on the dominant side and patients who had not experienced shoulder pain or dysfunction in the shoulder on the non-surgery side among the patients who visited the outpatient clinic of the department of rehabilitation medicine 3-4 months after arthroscopic rotator cuff repair from June to December 2016. The exclusion criteria were as follows: patients who had undergone rotator cuff repair surgery for

Table 1. Baseline characteristics of participants $(n=34)$

\begin{tabular}{|cc}
\hline \multicolumn{1}{c}{ Characteristic } & Value \\
\hline Age (yr) & $59.1 \pm 7.4$ \\
$\quad$ Median (range) & $60(46-78)$ \\
\hline Sex & $12(35.29)$ \\
$\quad$ Male & $22(64.71)$ \\
$\quad$ Female & $98.1 \pm 7.7$ \\
\hline Postoperative day & $99.5(97-112)$ \\
$\quad$ Median (range) & $12(35.29)$ \\
\hline Tendon tear type & $22(64.71)$ \\
$\quad$ Full thickness & \\
$\quad$ Partial thickness & $27(79.41)$ \\
\hline Surgery side & $7(20.59)$ \\
$\quad$ Right & $16.3 \pm 7.8$ \\
$\quad$ Left & $12(6-36)$ \\
\hline $\begin{array}{l}\text { Time from symptom onset to } \\
\text { operation (mo) } \\
\quad \text { Median (range) }\end{array}$ & \\
\hline
\end{tabular}

Values are presented as mean \pm standard deviation or number (\%). a muscle other than the supraspinatus muscle, patients who had undergone surgery for both shoulders, patients who had shoulder trauma history, and patients with an unclear muscle boundary on an ultrasonography image.

\section{Ultrasonography assessment}

Ultrasonography was performed by two researchers with a 5.0- to 12.0-MHz linear array probe (HD11 XE ultrasound system; Philips Healthcare, Best, The Netherlands). Researcher 1 was a rehabilitation medicine specialist with 10 years of experience performing ultrasonography for musculoskeletal diseases. Researcher 2 was a rehabilitation medicine resident with 2 years of experience performing ultrasonography for musculoskeletal diseases.

The maximal CSA of the muscle is closely related to muscle function [14]. Yanagisawa et al. [15] demonstrated that the maximal CSA of the supraspinatus muscle can be measured slightly lateral to the center of the scapula with MRI and that the CSA is not significantly different around the center of the scapula. There is no gold standard for positioning the ultrasound probe when measuring the CSA of the supraspinatus muscle; but, there are two studies in which reliability has been confirmed [16]. The first method to measure the CSA is as follows. The most lateral edge of the scapula spine and coracoid process are confirmed, and a straight line connecting the two landmarks is drawn. At the midpoint of the straight line, a

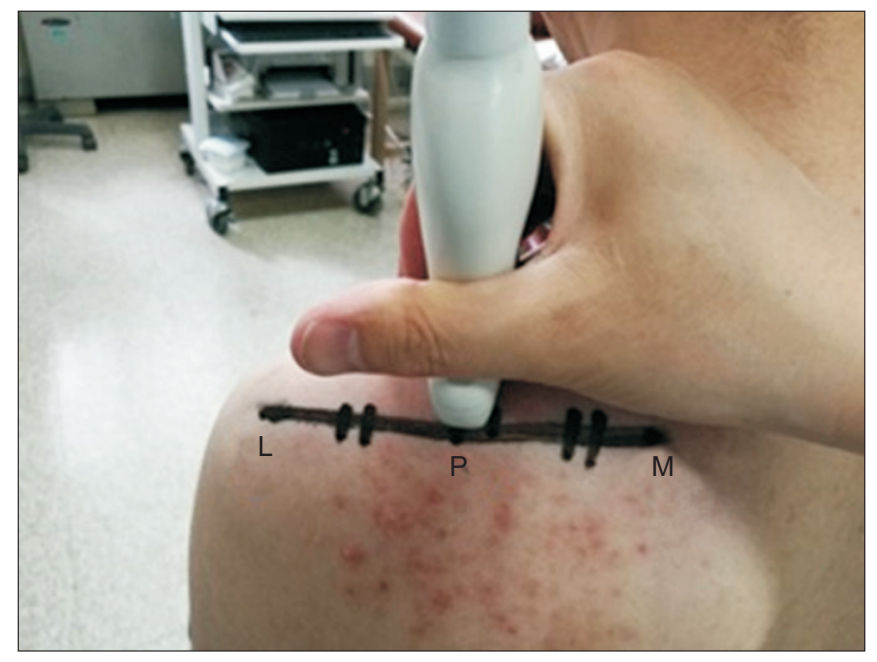

Fig. 1. Measurement location of the supraspinatus muscle cross-sectional area (CSA). The CSA was measured by placing the lateral edge of the probe on $\mathrm{P}, \mathrm{M}$, and most medial edge of scapula spine. $L$, most lateral edge of scapula spine; $\mathrm{P}$, the center of the line connecting $\mathrm{M}$ with $\mathrm{L}$. 
vertical line is drawn, and the CSA is measured in the site where the scapular notch is seen by moving the probe of the ultrasound along the vertical line [17]. The second method is as follows. The most lateral and medial edges of the scapular spine are confirmed by palpitation, and a straight line connecting the two points is drawn, the CSA is then measured at the midpoint of the straight line [18]. In this study, the CSA was measured using the second method, because the CSA is measured at the center of the scapula, which is close to the where the maximal CSA can be measured and easy to confirm with the surface anatomy.

To identify intra- and inter-rater reliability, for the first 14 of 34 subjects, two researchers checked the CSA of the supraspinatus muscle twice, respectively, and saved the images. For the remaining 20 subjects, Researcher 2 checked the CSA of the supraspinatus muscle and saved the images. For the first 14 of the 34 subjects, ultrasonography was conducted at the patient's outpatient clinic visit 3-4 months after arthroscopic rotator cuff repair, and again 2-3 days later at the patient's physical therapy session. The subjects were instructed to sit and naturally lower their arms with their elbows extended in a neutral position without supination or pronation of the forearm $[16,17]$. To capture the CSA images, the most lateral and medial edges of the scapular spine were assessed by palpitation, and a straight line connecting the two points was drawn. After confirming the CSA by vertically plac-
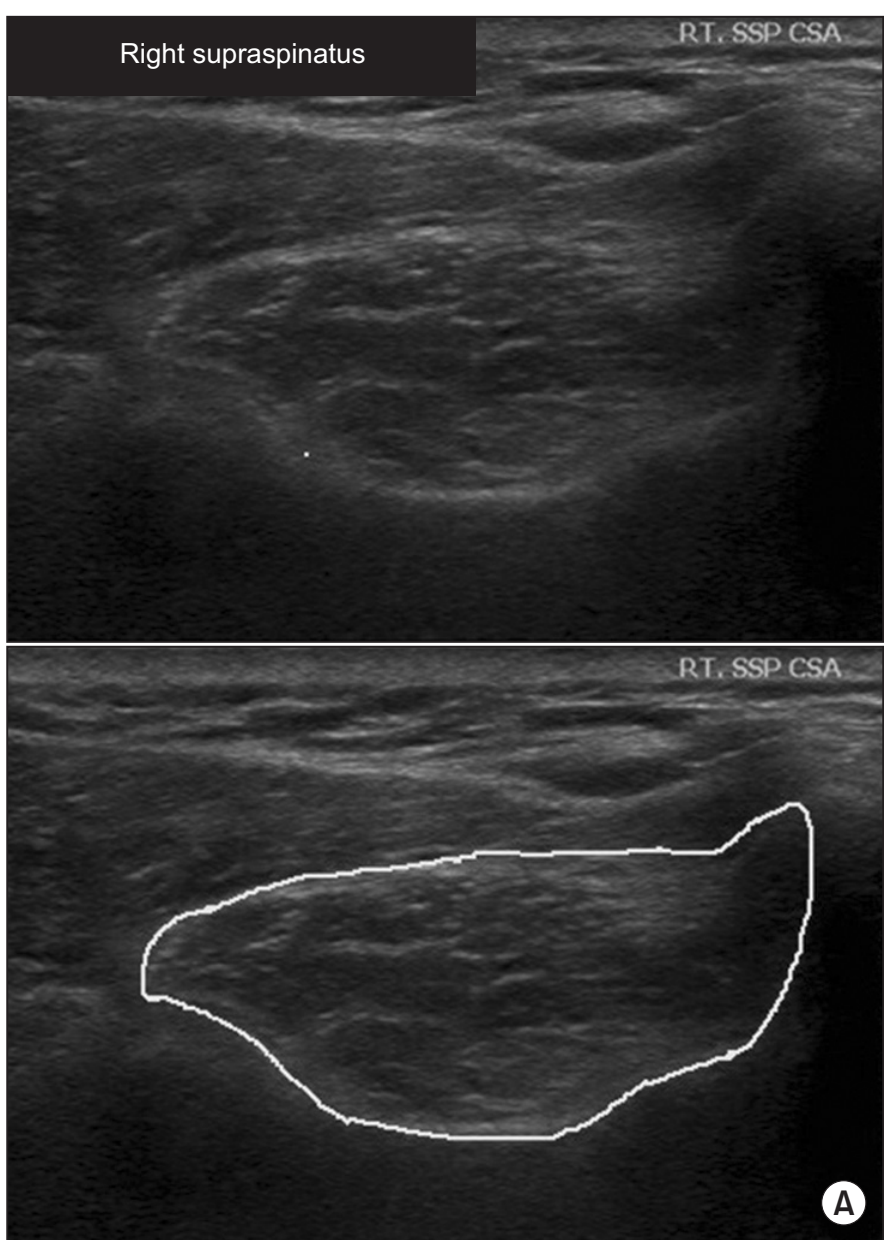
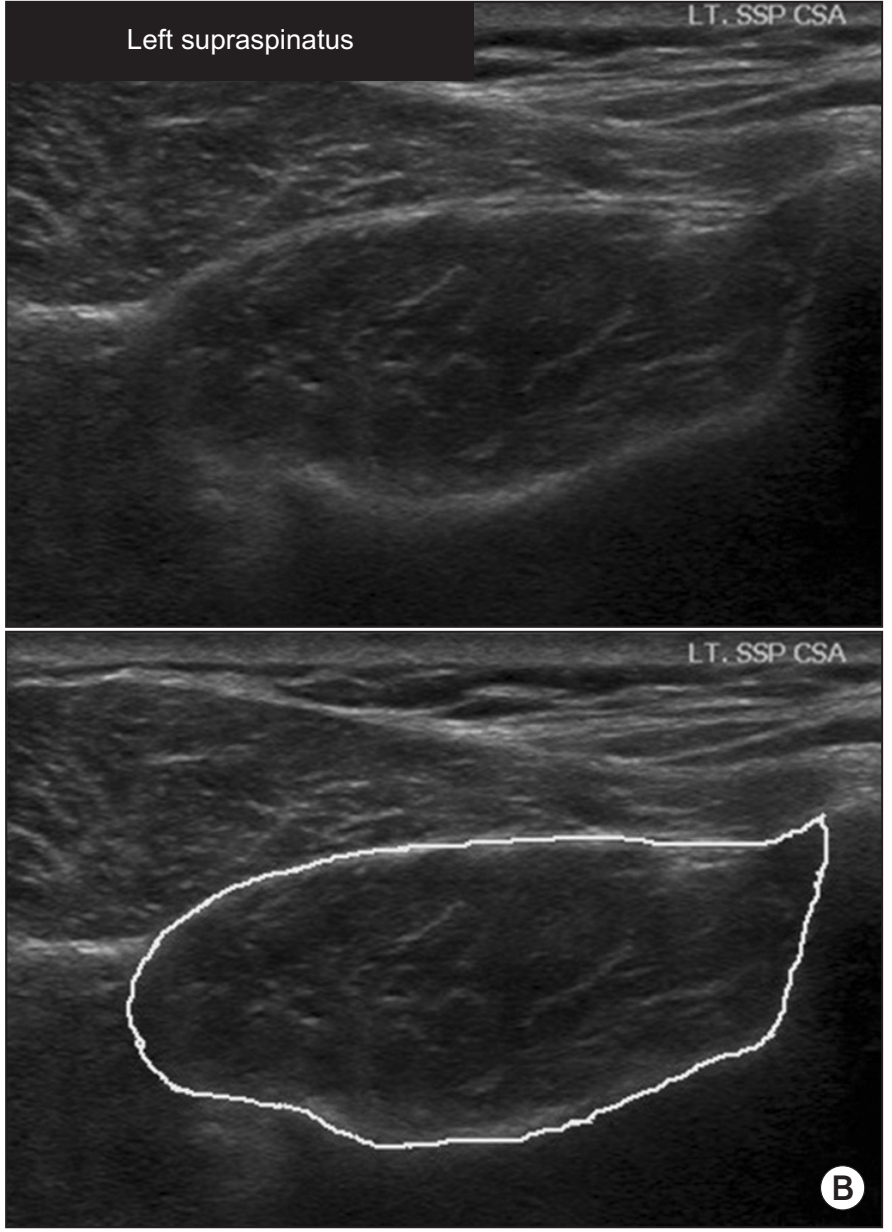

Fig. 2. Ultrasonography evaluation 3 months after arthroscopic right supraspinatus muscle tendon repair. (A) Right supraspinatus muscle (cross-sectional area $[\mathrm{CSA}]=377.72 \mathrm{~mm}^{2}$, mean echogenicity=76.39). (B) Left supraspinatus muscle $\left(\mathrm{CSA}=479.07 \mathrm{~mm}^{2}\right.$, mean echogenicity $\left.=63.89\right)$. Top image is the original image. Bottom image is the bordered image that was used for measurement. Supraspinatus CSA ratio (\%): $\frac{377.72 \text { (surgery side) }}{479.07 \text { ( }} \times 100=78.84$. Echogenicity ratio (\%): $\frac{76.39 \text { (surgery side) }}{63.89 \text { (non-surgery side) }} \times 100=119.56$. 
ing the probe of the ultrasound at the midpoint of the straight line, the image was saved (Fig. 1). The CSA and echogenicity in the saved images were measured using an image processing software program (Philips QLap); the CSA and mean echogenicity were calculated when the CSA boundaries of the saved images were specified. A third researcher who was not involved in the ultrasonography and did not have knowledge of patient information drew the CSA boundaries of the saved images (Fig. 2).

\section{Functional assessment}

The active ROM of the shoulder on the surgery side was measured and pain was assessed using the visual analogue scale (VAS). A questionnaire regarding the constant score of the shoulder was used, which assessed pain, activities of daily living, ROM and abduction strength of the shoulder joint. For the shoulder constant score, the shoulder abduction strength item was excluded because many patients complained of pain during the strength measurement, which made it difficult to obtain accurate measurements. Therefore, 75 was considered the highest score instead of 100 (excluding 25 for the strength measurement) for the shoulder constant score.

\section{Statistical analysis}

Using the intraclass correlation coefficient, intra- and inter-rater reliability were assessed according to the values of the CSA and echogenicity of the supraspinatus muscle as measured using ultrasonography for the initial 14 patients. Differences in the CSA and echogenicity between the surgery and non-surgery sides were compared using the Wilcoxon signed-rank test. Associations among the CSA, echogenicity, shoulder ROM, VAS, and constant score were identified using the Spearman rank correlation coefficients. The problem of multiple comparisons was adjusted for by using the Holm-Bonferroni method. The CSA and echogenicity of the full-thickness and partial-thickness tear groups were compared using the Wilcoxon rank sum test. All statistical analyses were performed using the SAS version 9.3 software (SAS Institute Inc., Cary, NC, USA). A significance level of $\mathrm{p}<0.05$ was used for all comparisons.

\section{RESULTS}

High intra- and inter-rater reliability were confirmed for the CSA and echogenicity measures (Table 2). There were statistically significant differences in the CSA and echogenicity between the surgery and non-surgery sides (Table 3). Regarding the associations of shoulder function with the CSA and echogenicity ratios that represented the degrees of muscle atrophy and fat infiltration, respectively, the CSA ratio was associated with shoulder forward flexion, external rotation, and constant score; however, the echogenicity ratio was not associated with shoulder function after surgery (Table 4). For the comparison of

Table 2. Intra- and inter-rater reliability of the supraspinatus muscle CSA and echogenicity measurement using ultrasonography

\begin{tabular}{cccccc}
\hline & \multicolumn{2}{c}{ CSA } & & \multicolumn{2}{c}{ Echogenicity } \\
\cline { 2 - 3 } \cline { 5 - 5 } & Researcher 1 & Researcher 2 & & Researcher 1 & Researcher 2 \\
\hline Intra-rater reliability & $0.93(0.8-0.98)$ & $0.96(0.89-0.99)$ & & $0.95(0.87-0.99)$ & $0.98(0.93-0.99)$ \\
Inter-rater reliability & \multicolumn{2}{c}{$0.98(0.95-0.99)$} & & $0.98(0.95-0.99)$ \\
\hline
\end{tabular}

Values are presented as intraclass correlation coefficient (95\% confidence interval).

CSA, cross-sectional area.

Table 3. Comparing the SSP CSA and echogenicity between the surgery and non-surgery sides

\begin{tabular}{lccc}
\hline & Surgery side & Non-surgery side & p-value \\
\hline SSP CSA & $532.4 \pm 132.8$ & $596.1 \pm 119.4$ & $<0.001^{* *}$ \\
Echogenicity & $65.3 \pm 14.4$ & $58.7 \pm 13.7$ & $<0.001^{* *}$ \\
\hline
\end{tabular}

Values are presented as mean \pm standard deviation.

The data in the table are calculated values of the images captured by Researcher 2 .

SSP, supraspinatus muscle; CSA, cross-sectional area.

${ }^{* *} \mathrm{p}<0.01$ by Wilcoxon signed-rank test. 
Table 4. Correlation coefficients between the SSP CSA ratio, echogenicity ratio, and shoulder function

\begin{tabular}{|c|c|c|c|c|c|c|}
\hline & \multicolumn{4}{|c|}{ Shoulder active ROM } & \multirow[b]{2}{*}{ Pain (VAS) } & \multirow[b]{2}{*}{$\begin{array}{l}\text { Constant } \\
\text { score }\end{array}$} \\
\hline & Flexion & Abduction & $\begin{array}{l}\text { Internal } \\
\text { rotation }\end{array}$ & $\begin{array}{l}\text { External } \\
\text { rotation }\end{array}$ & & \\
\hline SSP CSA ratio & $0.55\left(0.04^{*}\right)$ & $0.47(0.186)$ & $0.24(>0.999)$ & $0.65\left(0.002^{* *}\right)$ & $-0.45(0.269)$ & $0.57\left(0.025^{*}\right)$ \\
\hline Echogenicity ratio & $-0.52(0.072)$ & $-0.45(0.299)$ & $-0.26(>0.999)$ & $-0.42(0.422)$ & $0.5(0.101)$ & $-0.53(0.066)$ \\
\hline
\end{tabular}

Values are presented as Spearman correlation coefficient ( $\mathrm{p}$-value).

The data in the table are calculated values of the images captured by Researcher 2.

SSP, supraspinatus; CSA, cross-sectional area; ROM, range of motion; VAS, visual analogue scale.

${ }^{*} \mathrm{p}<0.05$ and ${ }^{* *} \mathrm{p}<0.01$ by Spearman correlation. Adjustment for the problem of multiple comparisons using the Holm-

Bonferroni method.

Table 5. Comparing the SSP CSA ratio and echogenicity ratio based on the supraspinatus muscle tendon tear type

\begin{tabular}{lccc}
\hline & Full thickness tear & Partial thickness tear & p-value \\
\hline SSP CSA ratio (\%) & $84.1 \pm 10.3$ & $92.2 \pm 12.9$ & 0.157 \\
\hline Echogenicity ratio (\%) & $114.4 \pm 13.9$ & $110.7 \pm 9.7$ & 0.327 \\
\hline
\end{tabular}

Values are presented as mean \pm standard deviation.

The data in the table are calculated values of the images captured by Researcher 2 .

SSP, supraspinatus muscle; CSA, cross-sectional area.

${ }^{*} \mathrm{p}<0.05$ by Wilcoxon rank sum test.

SSP CSA ratio $(\%)$ and echogenicity ratio $(\%)=\frac{\text { surgery side }}{\text { non-surgery side }} \times 100$.

the full-thickness and partial-thickness tear groups, there was no statistically significant difference between groups (Table 5). There was no association between the CSA and echogenicity ratios (correlation coefficients $=-0.43$, $\mathrm{p}=0.359$ ).

\section{DISCUSSION}

With the increased interest in the quantitative measurement of muscle morphology, more studies on the area have been published. Lee et al. [19] demonstrated that the degree of fat infiltration of the rotator cuff muscle as measured by quantitative and qualitative methods using CT arthrography are correlated and the reliability of the quantitative method is higher. However, there were disadvantages to the study of Lee et al. [19] in that MRI was used as a standard protocol for RCT evaluation rather than CT; the Hounsfield unit (HU) that was used for quantitative measurement of fat infiltration is not a direct measurement of the muscle lipid content; and radiation exposure was inevitable. Matsumura et al. [20] demonstrated that quantitative measurement of fat infiltration and atrophy of the whole muscle, not just the CSA of the muscle, is possible using three-dimensional, 2-point Dix- on MRI. However, there were several limitations to the study by Matsumura et al. [20]. Because the technology has not yet been sufficiently developed, the boundaries should be manually marked on all image slides, which is time consuming. Moreover, although there were no differences in the weight or height between the groups, the muscle morphology may differ depending on age or sex, even for persons with a similar physique [21]. Thus, it is difficult to accurately determine the state of the muscle by measuring the affected side only. With a simple method using ultrasonography, this study quantitatively evaluated the degrees of atrophy and fat infiltration of the supraspinatus muscle.

There were statistically significant differences in the CSA and echogenicity of the supraspinatus muscles between the surgery and non-surgery sides 3-4 months after arthroscopic rotator cuff repair. Because the ROM exercises were primarily performed up to 3 months after surgery while restricting strengthening exercises of the rotator cuff muscles, muscle atrophy and fat infiltration on the surgery side had predictable outcomes. However, various outcomes have been reported regarding muscle atrophy and fat infiltration after rotator cuff repair. One study reported that, after a 6-month and 1-year follow- 
up, the morphological changes of muscle are irreversible; once muscle morphology is changed, it cannot be recovered $[22,23]$. Another study reported that recovery from muscle atrophy is possible when rotator cuff repair is performed effectively [24,25]. Improvements in atrophy and fat infiltration of muscle after rotator cuff repair remain controversial.

Many studies demonstrated that the morphological changes of the muscle are associated with the occurrence of tendon tear or shoulder function $[23,26]$. However, only the rotator cuff tendon or tendon periphery were mainly evaluated when examining the shoulder with ultrasonography. This study evaluated the muscle itself with ultrasonography, and, because there was an association between the morphological changes of the muscle and shoulder function, this study demonstrated that rotator cuff muscle function could be predicted by evaluating the morphological changes of the muscle. Furthermore, this study demonstrated that objective evaluation is possible through quantitative measurement by using the symmetricity of the human body.

Although ultrasonography has the disadvantage of being a subjective (examiner-dependent) method, when fat infiltration was evaluated using the Goutallier grading system and muscle atrophy was evaluated using the tangent sign based on shoulder MRI by orthopedic specialists, the inter-observer agreement of the Goutallier grading system was 0.36 and that of the tangent sign was 0.59 , which indicates that the inter-observer consistency was low [27]. Both the Goutallier grading system and tangent sign are qualitative evaluation methods, and their results suggest that objective evaluation through quantitative measurement is more important than the type of examination.

In another study, fat infiltration was evaluated using ultrasonography; the degree of fat infiltration was evaluated by comparing the echogenicity of the supraspinatus muscle with that of subcutaneous fat or the trapezius muscle [8-10]. However, hypertrophy of the muscles around the supraspinatus muscle may occur as compensation or fat infiltration may progress together in the muscles around the supraspinatus muscle on the injured side; therefore, this evaluation method may be inappropriate [28]. Because the evaluation was performed based on the non-surgery side using the symmetricity of the human body, this study is superior to other similar studies. In addition, examining the non-surgery side with MRI is economically burdensome, and CT has the disadvantage of radiation exposure. Therefore, it is difficult to evaluate the non-surgery side with MRI or CT. Evaluation through ultrasonography can be performed immediately in the treatment room, is not particularly harmful to the patient, and has a minimal economic burden. Therefore, ultrasonography is an optimal examination tool for the evaluation of the morphological changes of the muscle using the symmetricity of the human body. Moreover, muscle atrophy and fat infiltration were reported to be associated with poor prognosis because it is not possible to recover even after surgery $[26,29,30]$. If fat infiltration or atrophy is severe on the injured side compared to the healthy side on preoperative ultrasonography, then rapid surgery may be recommended to prevent exacerbation of the unrecovered muscle atrophy or fat infiltration.

In this study, there was no association between the degree of supraspinatus muscle atrophy and fat infiltration. The association between the degree of muscle atrophy and fat infiltration are still controversial. Because the measurement methods for muscle atrophy and fat infiltration were not unified in each study, the degree of fat infiltration was not evaluated quantitatively [3133]. Moreover, when comparing the fat infiltration of the supraspinatus muscle as determined by MRI and ultrasonography, it is known that ultrasonography cannot distinguish between moderate and severe fat infiltration [33].

Therefore, muscle atrophy rather than fat infiltration can be considered a more function-related and objective evaluation index in the evaluation of the muscle morphology using ultrasonography. For this reason, in this study, it was considered that there was some association between the CSA ratio and shoulder function; but, there was no association between the echogenicity ratio and shoulder function, and between the echogenicity and CSA ratios.

In this study, the reliability was significantly higher compared to other studies and, unlike other studies, the inter-rater reliability was higher than the intra-rater reliability. The possible reason for the high reliability may be that the saved images were measured solely by a third researcher who was not involved in the ultrasonography. Had the researcher who saved the image directly measured the CSA using the image tool, it would have been possible to know which image was the surgical side, 
which may have affected the measured CSA value. As a result, since the reliability was significantly higher compared to other ultrasonography studies and the identified reliability itself was very high, it was thought that a higher inter-reliability would result than intra-rater reliability.

The supraspinatus CSA ratio was correlated with shoulder flexion and external rotation ROM; yet, it was not correlated with shoulder abduction ROM, which is the function of the supraspinatus muscle. The functional recovery of the shoulder is known to begin 3 months after rotator cuff repair and to continue as late as 12 months after the surgery [34]. This study included patients who underwent surgery 3 to 4 months prior. Thus, the lack of a significant correlation between the shoulder abduction ROM and supraspinatus CSA ratio may have been due to the functional recovery just beginning and/or limits to the ROM due to postoperative adhesion by disuse after the surgery.

This study had some limitations. First, the sample size was small. Second, although many studies have reported no significant differences in the evaluation of muscle atrophy and fat infiltration with ultrasonography and MRI, the values obtained by MRI and ultrasonography for the subjects who were involved in this study were not compared. In addition, many patients complained of pain during the strength measurement, which made it difficult to obtain accurate measurements. Therefore, the shoulder abduction strength item was excluded from the shoulder constant score. Another evaluation method for shoulder function will be necessary in future studies. Furthermore, most of the patients in this study did not undergo preoperative ultrasonography; consequently, it was impossible to make comparisons with their preoperative status. In addition, for the first 14 of 34 subjects, two researchers conducted the ultrasonography to check the reliability, and as the reliability was confirmed to be high, Researcher 2 conducted ultrasonography for the remaining 20 subjects. Although the reliability was high, there is a limitation in that statistical analysis was performed with calculated values of the images captured by Researcher 2, who was less experienced.

In this study, the potential of ultrasonography as an objective method of examination was confirmed through quantitative measurement based on the non-surgery side of the human body. Ultrasonography is the most harmless to patients and an accurate assessment method that is less economically burdensome. Moreover, it can be easily and immediately performed in the treatment room. However, it has been suggested that ultrasonography may be examiner-dependent and subjective. To overcome the subjectivity of ultrasonography and increase its objectivity, quantitative measurement studies need to be conducted continuously.

\section{CONFLICT OF INTEREST}

No potential conflict of interest relevant to this article was reported.

\section{ACKNOWLEDGMENTS}

The statistical consultation was supported by a grant of the Korea Health Technology R\&D Project through the Korea Health Industry Development Institute (KHIDI), funded by the Ministry of Health \& Welfare, Republic of Korea (Grant No. HI14C1062). This study was supported by the Sol Hospital research grant of the Korean Academy of Rehabilitation Medicine for 2016.

\section{REFERENCES}

1. Tashjian RZ. Epidemiology, natural history, and indications for treatment of rotator cuff tears. Clin Sports Med 2012;31:589-604.

2. Boszotta H, Prunner K. Arthroscopically assisted rotator cuff repair. Arthroscopy 2004;20:620-6.

3. Buess E, Steuber KU, Waibl B. Open versus arthroscopic rotator cuff repair: a comparative view of 96 cases. Arthroscopy 2005;21:597-604.

4. Charousset C, Grimberg J, Duranthon LD, Bellaiche L, Petrover D, Kalra K. The time for functional recovery after arthroscopic rotator cuff repair: correlation with tendon healing controlled by computed tomography arthrography. Arthroscopy 2008;24:25-33.

5. Goutallier D, Postel JM, Bernageau J, Lavau L, Voisin MC. Fatty muscle degeneration in cuff ruptures: preand postoperative evaluation by CT scan. Clin Orthop Relat Res 1994;304:78-83.

6. Jost B, Pfirrmann CW, Gerber C, Switzerland Z. Clinical outcome after structural failure of rotator cuff repairs. J Bone Joint Surg Am 2000;82:304-14.

7. Liem D, Lichtenberg S, Magosch P, Habermeyer P. 
Magnetic resonance imaging of arthroscopic supraspinatus tendon repair. J Bone Joint Surg Am 2007;89: 1770-6.

8. Khoury V, Cardinal E, Brassard P. Atrophy and fatty infiltration of the supraspinatus muscle: sonography versus MRI. AJR Am J Roentgenol 2008;190:1105-11.

9. Wall LB, Teefey SA, Middleton WD, Dahiya N, StegerMay K, Kim HM, et al. Diagnostic performance and reliability of ultrasonography for fatty degeneration of the rotator cuff muscles. J Bone Joint Surg Am 2012;94: e83.

10. Watanabe T, Terabayashi N, Fukuoka D, Murakami H, Ito $\mathrm{H}$, Matsuoka T, et al. A pilot study to assess Fatty infiltration of the supraspinatus in patients with rotator cuff tears: comparison with magnetic resonance imaging. Ultrasound Med Biol 2015;41:1779-83.

11. Katayose M, Magee DJ. The cross-sectional area of supraspinatus as measured by diagnostic ultrasound. J Bone Joint Surg Br 2001;83:565-8.

12. Karthikeyan S, Rai SB, Parsons H, Drew S, Smith CD, Griffin DR. Ultrasound dimensions of the rotator cuff in young healthy adults. J Shoulder Elbow Surg 2014;23:1107-12.

13. Reimers K, Reimers CD, Wagner S, Paetzke I, Pongratz DE. Skeletal muscle sonography: a correlative study of echogenicity and morphology. J Ultrasound Med 1993;12:73-7.

14. De Ste Croix M, Deighan M, Armstrong N. Assessment and interpretation of isokinetic muscle strength during growth and maturation. Sports Med 2003;33:72743.

15. Yanagisawa O, Dohi M, Okuwaki T, Tawara N, Niitsu M, Takahashi H. Appropriate slice location to assess maximal cross-sectional area of individual rotator cuff muscles in normal adults and athletes. Magn Reson Med Sci 2009;8:65-71.

16. Papatzika F, Papandreou M, Ekizos A, Panteli C, Arampatzis A. Reliability and limits of agreement of the supraspinatus muscle anatomical cross-sectional area assessment by ultrasonography. Ultrasound Med Biol 2015;41:1821-6.

17. Yi TI, Han IS, Kim JS, Jin JR, Han JS. Reliability of the supraspinatus muscle thickness measurement by ultrasonography. Ann Rehabil Med 2012;36:488-95.

18. Juul-Kristensen B, Bojsen-Moller F, Holst E, Ekdahl C. Comparison of muscle sizes and moment arms of two rotator cuff muscles measured by ultrasonography and magnetic resonance imaging. Eur J Ultrasound 2000;11:161-73.

19. Lee E, Choi JA, Oh JH, Ahn S, Hong SH, Chai JW, et al. Fatty degeneration of the rotator cuff muscles on pre- and postoperative CT arthrography (CTA): is the Goutallier grading system reliable? Skeletal Radiol 2013;42:1259-67.

20. Matsumura N, Oguro S, Okuda S, Jinzaki M, Matsumoto M, Nakamura M, et al. Quantitative assessment of fatty infiltration and muscle volume of the rotator cuff muscles using 3-dimensional 2-point Dixon magnetic resonance imaging. J Shoulder Elbow Surg 2017;26:e309-18.

21. Raz Y, Henseler JF, Kolk A, Riaz M, van der Zwaal P, Nagels J, et al. Patterns of age-associated degeneration differ in shoulder muscles. Front Aging Neurosci 2015;7:236.

22. Hata Y, Saitoh S, Murakami N, Kobayashi H, Kaito T, Kato H. Volume changes of supraspinatus and infraspinatus muscles after supraspinatus tendon repair: a magnetic resonance imaging study. J Shoulder Elbow Surg 2005;14:631-5.

23. Gladstone JN, Bishop JY, Lo IK, Flatow EL. Fatty infiltration and atrophy of the rotator cuff do not improve after rotator cuff repair and correlate with poor functional outcome. Am J Sports Med 2007;35:719-28.

24. Chung SW, Kim SH, Tae SK, Yoon JP, Choi JA, Oh JH. Is the supraspinatus muscle atrophy truly irreversible after surgical repair of rotator cuff tears? Clin Orthop Surg 2013;5:55-65.

25. Jo $\mathrm{CH}$, Shin JS. Cross-sectional area of the supraspinatus muscle after rotator cuff repair: an anatomic measure of outcome. J Bone Joint Surg Am 2013;95:178591.

26. Kuzel BR, Grindel S, Papandrea R, Ziegler D. Fatty infiltration and rotator cuff atrophy. J Am Acad Orthop Surg 2013;21:613-23.

27. Spencer EE, Dunn WR, Wright RW, Wolf BR, Spindler $\mathrm{KP}, \mathrm{McCarty} \mathrm{E}$, et al. Interobserver agreement in the classification of rotator cuff tears using magnetic resonance imaging. Am J Sports Med 2008;36:99-103.

28. Sofka CM, Lin J, Feinberg J, Potter HG. Teres minor denervation on routine magnetic resonance imaging of the shoulder. Skeletal Radiol 2004;33:514-8.

29. Uhthoff HK, Matsumoto F, Trudel G, Himori K. Early 
reattachment does not reverse atrophy and fat accumulation of the supraspinatus: an experimental study in rabbits. J Orthop Res 2003;21:386-92.

30. van de Sande MA, Stoel BC, Obermann WR, Tjong a Lieng JG, Rozing PM. Quantitative assessment of fatty degeneration in rotator cuff muscles determined with computed tomography. Invest Radiol 2005;40:313-9.

31. Kenn W, Bohm D, Gohlke F, Hummer C, Kostler H, Hahn D. 2D SPLASH: a new method to determine the fatty infiltration of the rotator cuff muscles. Eur Radiol 2004;14:2331-6.

32. Fuchs B, Weishaupt D, Zanetti M, Hodler J, Gerber C.
Fatty degeneration of the muscles of the rotator cuff: assessment by computed tomography versus magnetic resonance imaging. J Shoulder Elbow Surg 1999;8: 599-605.

33. Zanetti M, Gerber C, Hodler J. Quantitative assessment of the muscles of the rotator cuff with magnetic resonance imaging. Invest Radiol 1998;33:163-70.

34. Manaka T, Ito Y, Matsumoto I, Takaoka K, Nakamura $\mathrm{H}$. Functional recovery period after arthroscopic rotator cuff repair: is it predictable before surgery? Clin Orthop Relat Res 2011;469:1660-6. 